\title{
3D Printing of Reservoir Devices for Oral Drug Delivery: From Concept to Functionality through Design Improvement for Enhanced Mucoadhesion
}

Vaut, Lukas; Juszczyk, Julia J.; Kamguyan, Khorshid; Jensen, Kristian E.; Tosello, Guido; Boisen, Anja

Published in:

A C S Biomaterials Science \& Engineering

Link to article, DOI:

10.1021/acsbiomaterials.9b01760

Publication date:

2020

Document Version

Peer reviewed version

Link back to DTU Orbit

Citation (APA):

Vaut, L., Juszczyk, J. J., Kamguyan, K., Jensen, K. E., Tosello, G., \& Boisen, A. (2020). 3D Printing of Reservoir Devices for Oral Drug Delivery: From Concept to Functionality through Design Improvement for Enhanced Mucoadhesion. A C S Biomaterials Science \& Engineering, 6, 24782486.

https://doi.org/10.1021/acsbiomaterials.9b01760

\section{General rights}

Copyright and moral rights for the publications made accessible in the public portal are retained by the authors and/or other copyright owners and it is a condition of accessing publications that users recognise and abide by the legal requirements associated with these rights.

- Users may download and print one copy of any publication from the public portal for the purpose of private study or research.

- You may not further distribute the material or use it for any profit-making activity or commercial gain

- You may freely distribute the URL identifying the publication in the public portal 


\title{
3D Printing of Reservoir Devices for Oral Drug Delivery: FromConcept to Functionality through Design Improvement for Enhanced Mucoadhesion
}

\author{
Lukas Vaut,* Julia J. Juszczyk, Khorshid Kamguyan, Kristian E. Jensen, Guido Tosello, and Anja Boisen*
}

ABSTRACT: So far, microdevices for oral drug delivery have been fabricated as square or cylindrical reservoir structure $g^{\mathrm{a}}$ with a localized and unidirectional release. The fabrication is usually carried out using sophisticated and costly microfabrication techniques. Here, 3D printing of microreservoirs on sacrificial substrates is presented. This approach allows the devices to be accurately arranged in predetermined patterns, enabling implemen-tation into batch production schemes in which the fabrication of thedevices is linked to processing steps such as automated drug loading and sealing. Moreover, design and 3D printing of alternative geometries of minireservoirs featuring anchor-like surface structures for improved mucoadhesion and intestinal retention is demon- strated.

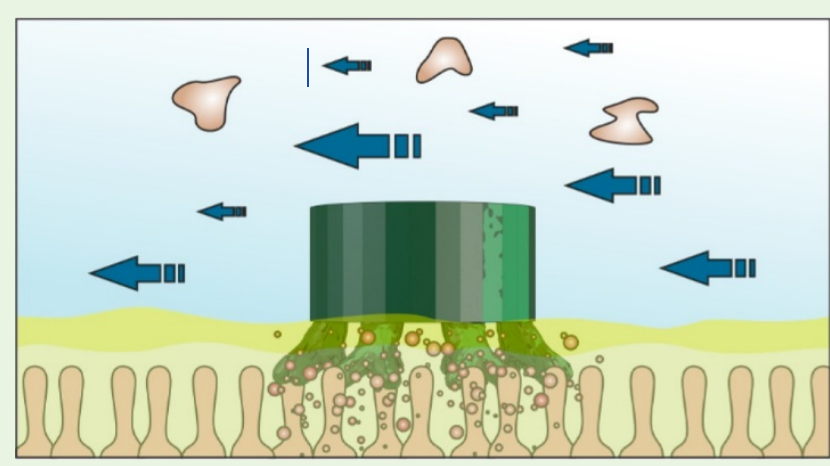
Surface texturing of minireservoirs increases mucoadhesion of the devices up to two-fold compared to a nonstructured control. The structuring also leads to a strong bias in mucoadhesion in different orientations, which can facilitate a correct orientation of the devices and thus lead to unidirectional release of drugs toward the intestinal mucosa for increased drug uptake.

KEYWORDS: micro additive manufacturing, oral drug delivery, microdevices, microcontainers, minireservoirs, mucoadhesion

Engineered microfabricated oral drug delivery (ODD) devices have emerged as an alternative strategy for increasing bioavailability. ${ }^{1,2}$ Since oral delivery is the preferred route of drug administration, much research effort has gone into the translation of parenteral administered dosage forms to orally administered dosage forms of drugs. ${ }^{3}$ This translation often faces difficulties, because many drugs exhibit poor bioavailability when administered orally, as the complex physiological environment of the gastro-intestinal tract causes degradation (gastric acid, digestive enzymes), prevention of absorption (mucus layer, epithelial barrier), and rapid clearance (mucus secretion, gastro-intestinal transit). To protect and transport drugs as well as to retain drugs close to their target site and promote absorption, various drug delivery technologies have been developed and investigated. Next to specialized coatings are particulate drug delivery systems relying on self-assembly of molecules and polymers, such as liposomes, nano/microparticles, etc. Microfabrication of ODD devices holds great potential for producing uniform devices with distinct morphologies, due to the involved top-down engineering process.

Initially, Ahmed and co-workers designed and fabricated microdevices for ODD as square, flat, and patch-like structures with a reservoir into which a drug can be filled. ${ }^{1}$ In contrast to particulate systems, microfabricated devices represent universal delivery platforms with the potential for a localized unidirectional release of the drug from the reservoir toward the target site. ${ }^{1}$ Furthermore, this effect can be promoted by specific chemical surface modification of the devices. ${ }^{1}$ On the basis of this concept, different fabrication protocols and materials have been demonstrated and the suitability of the devices for ODD was highlighted, e.g., through the fabrication and drug-filling of multilayered poly(methyl methacrylate), epoxy-based SU8, and biodegradable poly(DL-lactide-co-glycolide) microdevi- ces. $^{4}$ With respect to fabrication, the concept was furtherexpanded by the fabrication of biodegradable cylindrical microcontainers using hot punching, cylindrical SU8 micro- containers with $\mathrm{pH}$-sensitive lids made with photolithography, and capped microreservoir devices made by StampEd Assembly of polymer Layers (SEAL). ${ }^{5-7}$ In vitro as well as 
in vivo release studies have shown promising results for the drug delivery performance of microfabricated devices. ${ }^{6,8,9}$

Although Ahmed et al. and Chirra et al. demonstrated an increased association of lectin-functionalized microdevices with epithelial cells and enhanced intestinal retention in mice, respectively, the unidirectional release of the drug toward the target site was not facilitated or proven in the hitherto referred cases. $^{10,11}$ Furthermore, the employed fabrication techniques require elaborated lab conditions (e.g., clean room facilities) and are costly. 3D printing, on the contrary, is cheaper and generally considered to be a good tool for rapid prototyping, owing to a tool-free workflow and few design constraints. The production of prototypes with conventional technologies used for large scale production is often unfeasible as it is too expensive. ${ }^{12} 3 \mathrm{D}$ printing enables the customization of products as exemplified with the use of fused-deposition modeling (FDM) for personalized 3D printed dosage forms in the context of point-of-care medication. ${ }^{13,14}$ While print resolution limits FDM fabrication capabilities to the macroscale, vat photopolymerization-based 3D printing techniques, such as stereolithography (SL) or digital light processing (DLP) techniques, rely on operation principles that are very similar to those of photolithography. ${ }^{15}$ These techniques can thus produce mesoscale down to microscale components, making them suitable for prototyping of microdevices for ODD as lately demonstrated in a feasibility study making use of microSL. ${ }^{16}$ Although the latter mentioned study could showcase the use of micro-SL for the fabrication of different microreservoir devices for ODD, it also disclosed several weaknesses of the process. Those being a flawed cleaning protocol for the removal of uncured photopolymer from the fabricated devices, the inability to obtain predetermined patterns of microreservoir devices that are separable, and the use of costly machinery exhibiting a free surface configuration and having a low throughput. Nevertheless, 3D printing potentially offers much flexibility and sufficient throughput for fabrication of microfabricated ODD devices, enabling research and development of optimal shape and function.

In this work, we demonstrate the usage of a desktop DLP $3 \mathrm{D}$ printing system with a spatial resolution (i.e., voxel size) of $30 \mu \mathrm{m}$ and a constrained surface configuration to $3 \mathrm{D}$ print microreservoir devices for ODD in dimensions close to SU8 microcontainers fabricated previously. ${ }^{9}$ Moreover, the utilization of a recently developed 3D printing method potentially enables full integration into production schemes that combine device fabrication with further processing steps, such as drugloading and sealing. ${ }^{17}$ To showcase the prototyping potential of $3 \mathrm{D}$ printing for ODD devices, we present the $3 \mathrm{D}$ printing and characterization of miniaturized reservoir structures (minireservoirs) with different geometries designed to promote unidirectional release with controlled orientation and intestinal retention.

\section{MATERIALS AND METHODS}

2. I. Materials. All chemicals and reagents were used as-received. For the fabrication of sacrificial substrates from poly(vinyl alcohol) (PVA), RS Pro PVA 3D printing filament (RS Components A/S, Denmark) was used. HTM 140 M V2 3D printing photopolymer (EnvisionTEC GmbH, Germany) was used to 3D print reservoir structures. 2-Propanol (Sigma-Aldrich Denmark A/S, Denmark) was used for the post-treatment of 3D printed structures and Dulbecco's phosphate buffered saline (Sigma-Aldrich Denmark A/S, Denmark) was used in the flow retention mucoadhesion tests.
2.2. Computer Aided Design (CAD). All design tasks were carried out using OpenSCAD open source software and SolidWorks 2015 (Dassault Systens SolidWorks Corporation, U.S.A.).

2.3. Fabrication of Sacrificial PVA Substrates. The fabrication of PVA substrates was performed as previously described. ${ }^{17}$

2.4. 3D Printing of Reservoir Structures on Sacrificial PVA Substrates. Reservoir structures were 3D printed on top of PVA substrates as previously reported. ${ }^{17}$ After completion of the printing procedure, the substrate along with printed structures was sonicated in 2-propanol for $5 \mathrm{~min}$ to remove excess print material. After evaporation of the solvent, UV-postcuring was performed for $10 \mathrm{~min}$.

2.5. Release of 3D Prints from PVA Substrates. The PVA substrate along with printed structures was placed in a small sieve made from stainless steel filter mesh and inserted into a Milli-Q waterfilled beaker at a temperature of $55^{\circ} \mathrm{C}$ with magnetic stirring for a mild release of the structures.

2.6. Scanning Electron Microscopy. All scanning electron microscopy was performed using a TM3030Plus tabletop scanning electron microscope (Hitachi High Technologies Europe GmbH, Germany). A 208HR high resolution sputter coater (Cressington Scientific Instruments, U.K.) equipped with a gold target was used to coat the specimens with a thin layer of gold $(\sim 20 \mathrm{~nm})$ prior to observation.

2.7. Tensile Mucoadhesion Tests. Experiments were performed using a TA.XTplus texture analyzer (Stable Micro Systems Ltd., U.K.) equipped with a $500 \mathrm{~g}$ loadcell. The samples were fastened to the probe holder with use of $3 \mathrm{D}$ printed probes and double-sided adhesive facing downward and in the course of the experimentpressed down into a segment of porcine intestinal tissue, which was placed onto a holder platform with the mucosal side facing upward. After a contact time of $60 \mathrm{~s}$ and a contact force of $10 \mathrm{~g}$, according to intestinal contraction forces, the probe was moved upward at a speed

of $0.01 \mathrm{~mm} \mathrm{~s}^{-1} .18$ The experiments were performed in a replicated Latin square design with 5 different locations on two different tissues and the sequence of tested samples as blocking factors.

2.8. Flow Retention Mucoadhesion Tests. A flow retention setup was constructed from 3D printed parts. A segment of porcine intestinal tissue was placed on the slide with the mucosal side facing upward. The slide was held at an angle of $30^{\circ}$ relative to the horizontal plane and connected to the tubing of a 120S/DV peristaltic pump (Watson-Marlow Flexicon A/S, Denmark). To remove loose mucus, the tissue was initially flushed with Dulbecco's phosphate

buffered saline (PBS) at a flow rate of $40.9 \mathrm{~mL} \mathrm{~min}^{-1}$ for $5 \mathrm{~min}$. Ten samples were placed on the tissue either facing upward or downward without applying force and incubated on the tissue for $1 \mathrm{~min}$. Flow of PBS was started at a flow rate of $4.1 \mathrm{~mL} \mathrm{~min}^{-1}$ and increased with an

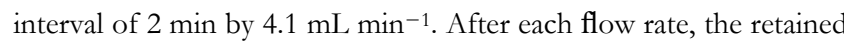
devices were counted. The experiment was performed in a Latin square design with 5 different tissue segments and sequence of tested devices as blocking factors for each orientation.

2.9. Simulations. Simulations were conducted using COMSOL Multiphysics 5.4 (COMSOL AB, Sweden). 3D designs of minireservoirs were imported and placed at the center of the wall inside a cylinder with a diameter of $50 \mathrm{~mm}$ (according to average human intestinal diameter) and a length of $100 \mathrm{~mm} .{ }^{19}$ Laminar flow conditions and physical properties of water were used for the simulations. The sizing of mesh elements was selectively chosen to be coarser for the tube and finer for the minireservoirs.

2.10. Statistics. All presented statistics were computed using $\mathrm{R}$ programming language and RStudio software (RStudio Inc., U.S.A.) as well as GraphPad Prism 7 (GraphPad Software, U.S.A.).

\section{RESULTS AND DISCUSSION}

3.I. 3D Printing of Microreservoirs on a Sacrificial Substrate. Bulk fabrication on a substrate (e.g., silicon wafer) is a common attribute of microdevices for ODD. Upon production completion, the individual devices are released by means of a sacrificial layer (e.g., water-soluble poly(acrylic acid) release layer) and harvested. ${ }^{9}$ Moreover, in micro- 


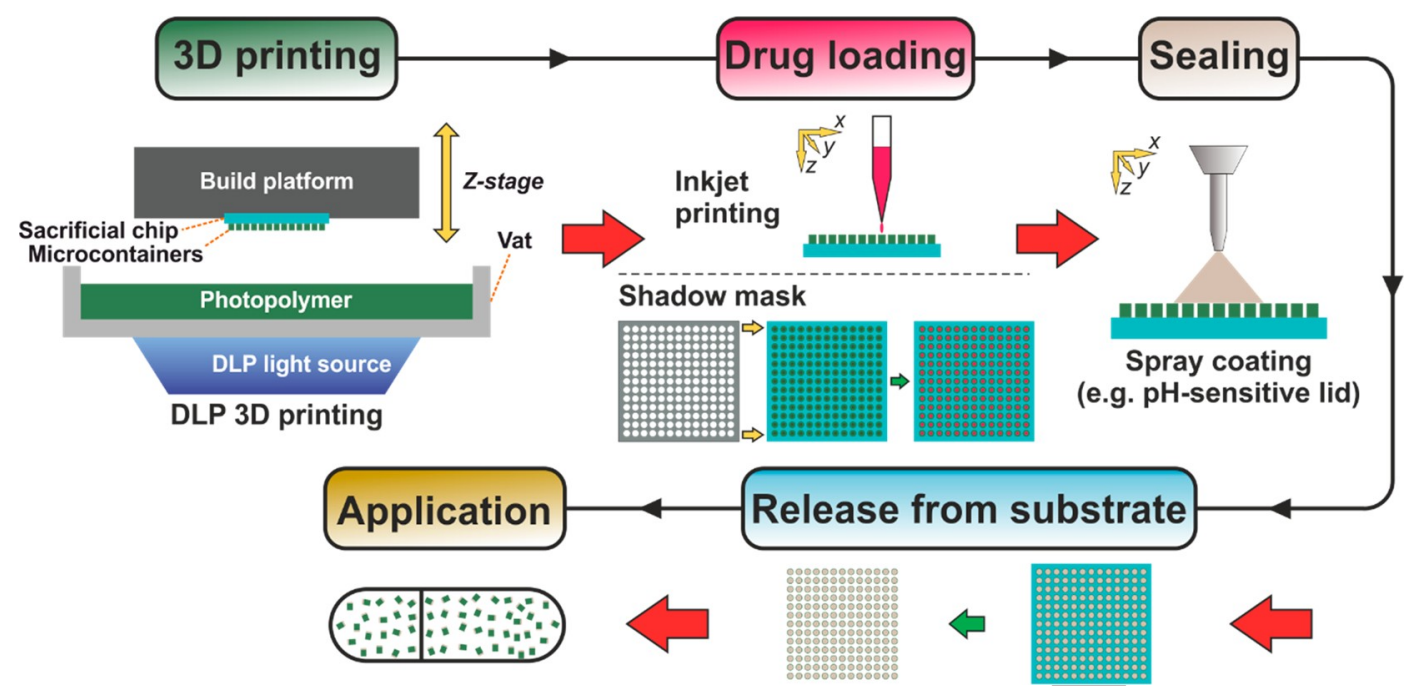

Figure 1. Workflow implementation of 3D printing into the fabrication of drug-loaded polymeric microreservoirs. 3D printing of arrays of micoreservoirs on a sacrificial polymer substrate using a specific 3D printing method enables the connection to further array-based processing steps that were previously demonstrated. ${ }^{17}$ For example drug loading via inkjet printing or shadow mask, subsequent sealing with, e.g., a $\mathrm{pH}$-sensitive lid via spray coating and finally release of single microreservoirs from sacrificial substrate and their harvesting for application. $1,6,9,20-22$

fabrication, the wafer connects multiple processing steps by preserving the patterning of structures throughout the entire process. In vat photopolymerization-based $3 \mathrm{D}$ printing, the structures are printed on a machine-specific build platform, which is precisely leveled to the polymerization interface of the vat. After the printing procedure has finished, the printed structures are usually manually removed from the platform, which is often facilitated with the use of hand tools such as pliers, scrapers, or razor blades. Finally, residual photopolymer is removed with a solvent. This process is, in principle, suitable for the fabrication of microdevices for ODD, however it prevents the utilization of subsequent processing steps in which the geometrical patterning of the devices is required. Previously, several techniques relying on geometrical patterning for the processing of microdevices for ODD were reported, for example the use of inkjet printing for drug loading, the application of micromachined shadow masks for drug loading, and lid sealing via spray coating. , $^{60-23} 3 \mathrm{D}$ printing microdevices with a likewise 3D printed base layer, thereby preserving the geometrical pattern (as done in a previous feasibility study ${ }^{16}$ ), is not a suitable option, because it irreversibly connects all devices to a whole and hence removes the possibility to release them individually.

The use of prefabricated water-soluble poly(vinyl alcohol) (PVA) substrates has recently been shown to enable the release of disconnected microstructures. The substrates can beinserted into a customized holder of a vat photopolymeriza- tion-based $3 \mathrm{D}$ printer and therein used as a $3 \mathrm{D}$ printing substrate. ${ }^{17}$ Translating this method to the $3 \mathrm{D}$ printing of microreservoir devices means that, similar to a wafer in microfabrication, the PVA substrates preserve the geometrical patterning of microreservoirs and thereby enable the connection of the fabrication method to further processing steps (Figures 1 and S1, Supporting Information, SI) such as drug loading and sealing. Finally, after completion of all processing steps, the substrate can be dissolved in water to release the individual microreservoirs, which can then be filledinto a capsule for oral dosage.

Using the aforementioned 3D printing technique, defined arrays of microreservoirs with different sizes and aspect-ratios
Figure 2). The printed microreservoirs had diameters of 570
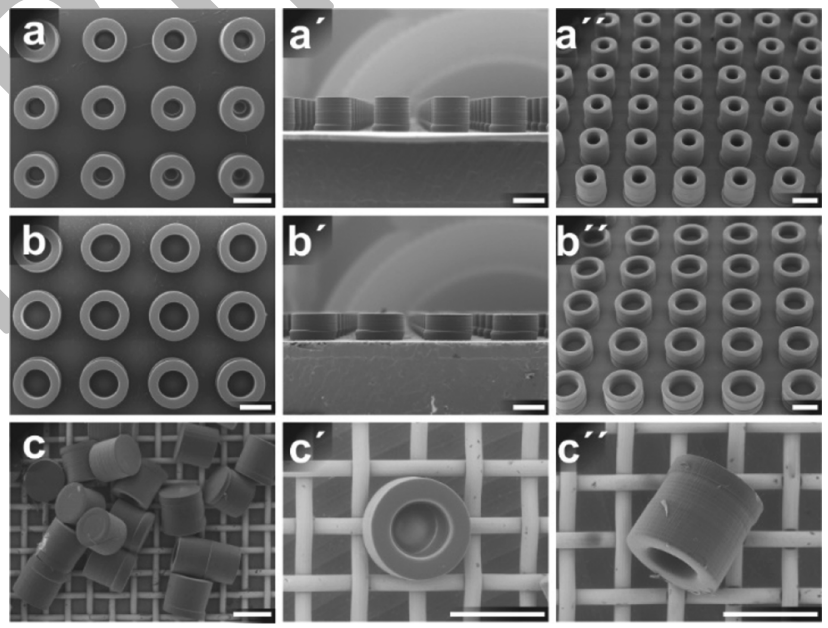

Figure 2. Scanning electron microscopy images of differently sized 3D printed microreservoirs. Scale bars are equivalent to $500 \mu \mathrm{m}$. (a) Microreservoirs with dimensions of $570 \times 660 \mu \mathrm{m}^{2}$ (diameter $\times$ height) and a wall thickness of $150 \mu \mathrm{m}$ on PVA substrate. (b) Microreservoirs with dimensions of $650 \times 350 \mu \mathrm{m}^{2}$ and a wall thickness of $130 \mu \mathrm{m}$ on PVA substrate. Top view, side view (') and $45^{\circ}$ tilted view ("). (c) Microreservoirs after release from sacrificial PVA substrate on steel filter mesh. Top view of several specimens, top view of single specimen (') and side view of single specimen (").

and $650 \mu \mathrm{m}$ with aspect-ratios of 1:1.16 and 1:0.54 (diameter/ height), respectively. The $3 \mathrm{D}$ printed microreservoirs had characteristics similar to those of SU8 microcontainers and remained intact after release from the sacrificial substrate.

The fabrication of cylindrical polymeric microreservoir devices as per the outlined $3 \mathrm{D}$ printing process chain is designed to be compatible with previously published postprocessing methods, such as drug loading using inkjet printing or shadow masks and sealing via spray coating. , $9,20,22^{2}$ Those methods were extensively described with respect to 

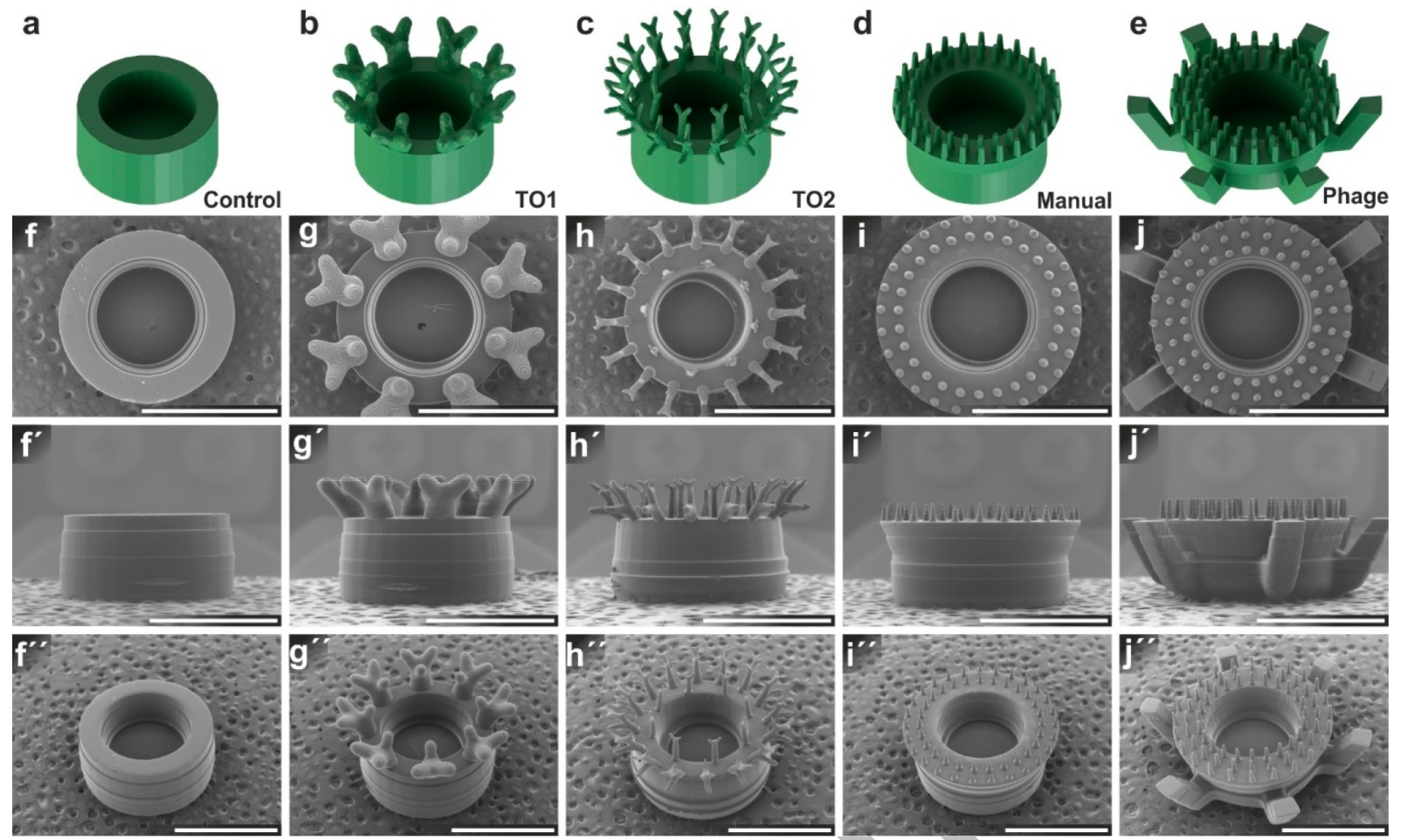

Figure 3. Design and fabrication of alternative reservoir geometries. (a, f) Plain reservoir structure as control specimen (“Control”). (b, g) Design with large branching edge anchors ("TO1"). (c, h) Design with small branching edge anchors ("TO2"). (d, i) Design featuring overhang and straight anchor spikes ("Manual"). (e, j) Bioinspired phage-style design with straight anchor spikes, rounded bottom side and legs ("Phage"). (a - e)3D design representations. $(\mathrm{f}-\mathrm{j})$ Scanning electron microscopy images of 3D printed structures. Scale bars correspond to $2 \mathrm{~mm}$. Top view, side view (') and $45^{\circ}$ tilted view (").

their functionality, i.e., loading capacity and in vitro release, in the respective publications and are not a subject of this work.

3.2. 3D Printing of Minireservoirs with Enhanced Mucoadhesion. One of the hypotheses related to microfabricated devices for ODD is that they facilitate a unidirectional release of the drug from the reservoir toward the target site, which in oral delivery most commonly is the intestinal mucosa. Unless the devices are self-propelling, their movement in the intestine and their orientation is entirely dependent on external forces, that in the intestinal environment are most likely due to intestinal motility and peristaltic flow of intestinal contents. In this case, the orientation of microdevices is presumably random if no bias is applied to their faces. Chirra et al. aimed at promoting unidirectional release toward the intestinal mucosa by designing microdevices with a flat aspectratio for decreased susceptibility to peristaltic shear stress and by functionalizing the reservoir side of the devices with lectins. ${ }^{11}$ The latter are a group of proteins that among others bind to carbohydrate chains present at the epithelial cell surface. ${ }^{24}$

3.2.I. Design. In contrast to such a chemistry-based approach, we present devices featuring a strong contrast between top and bottom geometry by means of specific surface texturing on the reservoir side (Figure $3 \mathrm{a}-\mathrm{e}$ ). According to the mechanical theory of mucoadhesion, the increase in surface area, and thereby increased surface interaction, enhances the "viscoelastic dissipation of energy during joint failure" and hence can increase adhesion to mucosal surfaces. ${ }^{25}$ The generated surface structures are intended to ease the penetration of the devices into the mucus layer by increasing the locally applied tension. Further, the increased friction forces between the mucus gel and the surface of the devices leads to enhanced detachment forces and thus stronger adhesion. The increased adhesiveness of the reservoir side compared to the bottom side of the devices has the potential to result in an orientation bias.

Next to a plain reservoir device ("Control"), two designs with different degrees of increased surface area ("TO1" and "TO2") were generated employing a topology optimization approach to solve a free form optimization problem. ${ }^{26}$ The topology optimization is not aimed at optimizing adhesion, but generates branching structures with large surface areas. Additionally, two more designs were manually created. The design named "Manual" features an overhang at the reservoir side (top side) as well as cone-shaped spikes for reduced fabrication complexity. This design is inspired by the overall shape of "TO1" and "TO2". The bioinspired "Phage"-design features an increased number of spikes, a rounded bottom side and side extensions along the lines of the morphology of T4 bacteriophages. The latter is known to infect bacteria by attaching to the cell surface through a sequential binding of the hexagonally arranged tail fibers, resulting in a stable upright position. ${ }^{27}$ It must be emphasized that all designs are exterior modifications to the same reservoir design with a fixed height and outer diameter.

3.2.2. 3D Printing. The presented designs were fabricated by $3 \mathrm{D}$ printing and characterized with scanning electron microscopy (Figure $3 \mathrm{f}-\mathrm{j}$ ). However, due to resolution limitations of the available $3 \mathrm{D}$ printing system, the size of the reservoirs was scaled up according to the minimum featuresize of the designs. Resolution arrays for the different designs (SI Figures S2-S5) were 3D printed to determine an 

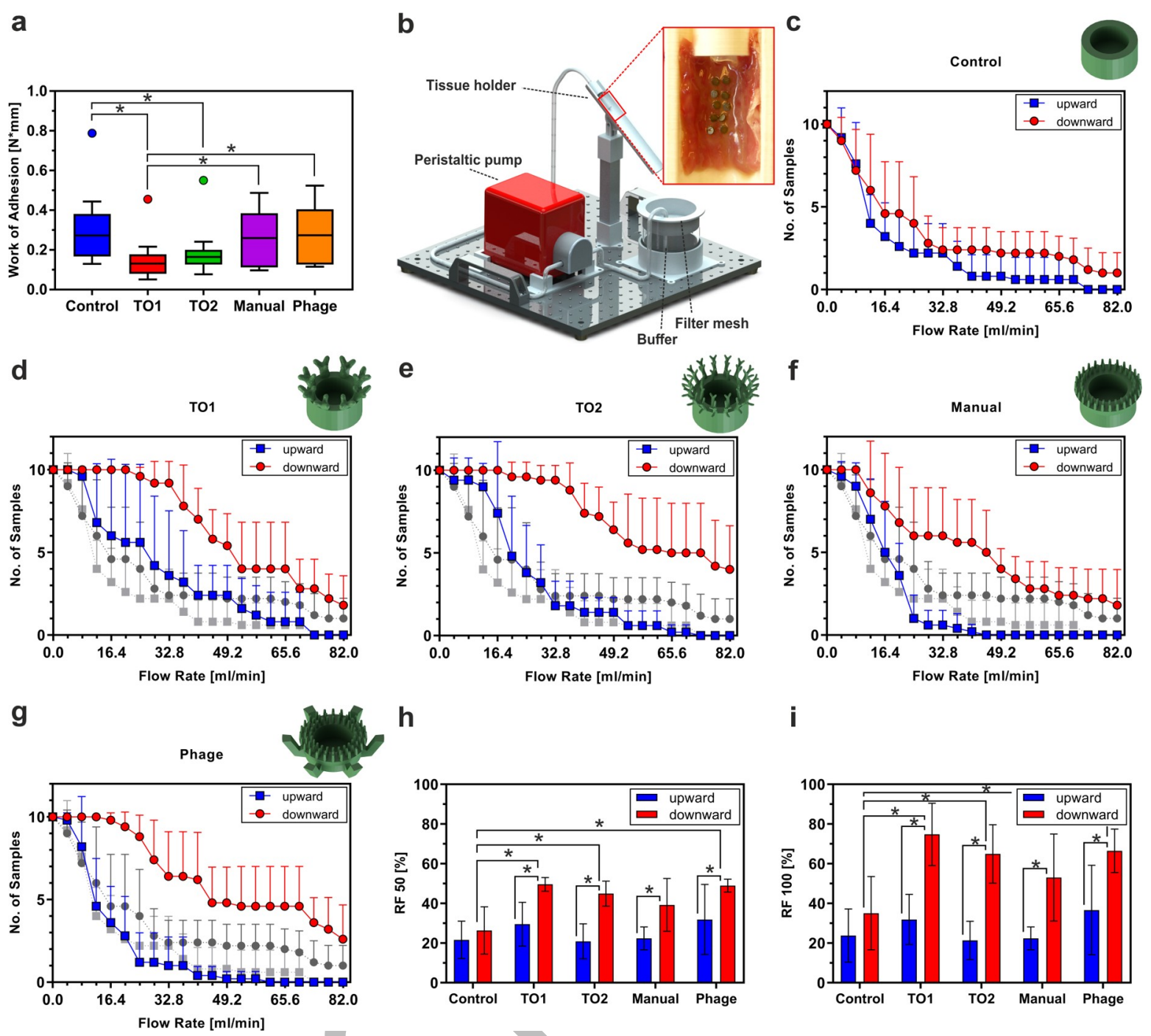

h

i
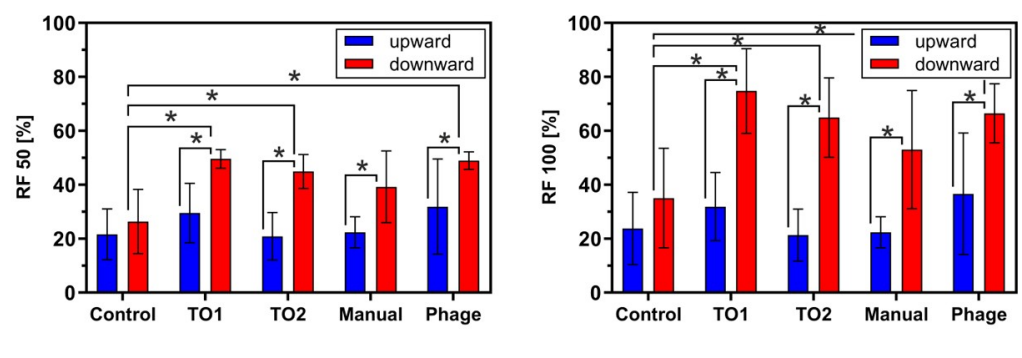

Figure 4. Characterization of mucoadhesion. (a) Analysis (Tukey boxplots) of mucoadhesion of alternative reservoir geometries with the tensile method. Work of adhesion (WOA) is defined as the area under the curve of the detachment graph where force is plotted against displacement $(N=$ 10). (b) Schematic of experimental setup for evaluation of mucoadhesion with the flow retention method adapted from Rao and Buri. ${ }^{30}$ Retention graphs and comparison between downward and upward orientation relative to mucosal surface for (c) "Control", (d) "TO1", (e) "TO2", (f) "Manual", and (g) "Phage" specimens. Values from c grayed out in $\mathrm{d}-\mathrm{g}$. Each flow rate lasted for 2 min $(N=5)$. (h) Comparison of RF 50 value between different designs and different orientations based on retention graphs. RF 50 is defined as the relative cumulative amount of retained specimens at 50\% of the maximally applied flow rate. (i) RF 100 values of different designs and orientations. RF 100 is defined like RF 50 , but at $100 \%$ of the maximum applied flow rate. Error bars represent $95 \%$ confidence intervals $(\mathrm{h}, \mathrm{i})$. Stars indicate statistical significance with $p<0.05$ according to Least Significant Difference test (a) and Welch's $t$ test (h, i).

achievable scale of the objects. In this aspect, "Manual" was not regarded, because it features the same dimensions as "Phage". Also, the spikes were disregarded as they were scaled independently from the overall size of the object. "TO2", with the smallest design elements, represents the size limiting factor. Consequently, a size with an outer reservoir diameter of $2.6 \mathrm{~mm}$ was chosen due to good repeatability. Using the same 3D printing method, also larger reservoir devices could be fabricated on sacrificial PVA substrates (SI Figure S6).

3.2.3. Tensile Mucoadhesion Study. Initially, the mucoadhesive effect of the surface texturing was tested with a tensile mucoadhesion setup and porcine intestinal tissue (SI Figure S7), thus simulating the effect of intestinal contraction events. The experiment was carried out in a replicated Latin square design with tissue as well as sequence of tested structures as blocking factors. This prevented the testing sequence and the variability of the tissue from affecting the analysis of the measurements. The comparison of work of adhesion (area under the curve of the detachment graph; Figure 4a) shows that samples "TO1" and "TO2" have significantly lower mucoadhesion with respect to "Control", whereas "TO1" has the lowest with also significantly lower values than "Manual" and "Phage". Analysis of maximum peak forces (SI Figure S7c) shows the same overall trend with the exception that "TO2" instead of "TO1" has the lowest values. In both cases, "Manual" and "Phage" are not significantly different from the control. While on the one hand it is expected that increased surface area in case of "TO1" and "TO2" leads to increased contact area, it can on the other hand be presumed that the branching surface structures result in a reduction of required penetration force and thus better penetration into the mucus. Due to the relatively high stiffness of the $3 \mathrm{D}$ printed structures, 
the vertical force applied from the tensile instrument, through the branching structures, probably induces higher shear stress to the mucus when compared to less penetrating structures. Since mucus is a shear-thinning gel, the consequent reduction in viscosity can lead to a decrease in detachment force. ${ }^{28}$ In this case, this method is not regarded as being suitable to evaluate the mucoadhesive potential of different designs with surface structures. Additionally, it has been pointed out that the occasions of tensile detachments are probably rare events from a physiological point of view. ${ }^{29}$ Therefore, the same minireservoirs were characterized for mucoadhesion using a flow retention setup (Figure $4 \mathrm{~b}$ ). ${ }^{30}$

3.2.4. Flow Retention Mucoadhesion Study. To prevent the influence of tissue variability and mucus integrity, the experiments were performed in a Latin square design with tissue and sequence of tested structures as blocking factors. In addition to comparing various designs, the initial orientation of the minireservoirs was included. Although the overall average flow rate in the human small intestine is reported to be up to 3 $\mathrm{mL} \mathrm{min}^{-1}$, the retention experiment was performed over a range of different flow rates $\left(4.1-81.9 \mathrm{~mL} \mathrm{~min}^{-1}\right)$, which might mimic the increased local shear stress induced by intestinal motility. ${ }^{31}$ Plotting the number of retained minireservoirs against the flow rates shows very diverging retention profiles for different designs and orientations (Figure $4 c-g)$. For comparison of the designs, the relative cumulative number of retained devices up to a flow rate corresponding to $50 \%$ of the used maximum flow rate (RF50) and up to the maximum used flow rate (RF100) were calculated and analyzed for statistical significance (Figure 4h, i). The results remarkably show that in both cases there is no significant difference between all samples when placed with the reservoir side upward. However, when placed downward on the surfaceof the intestinal tissue, all samples with surface structures show significantly higher values when compared to the control. Furthermore, all samples with surface structures, but not the control, show a significant effect of orientation. This suggests that the minireservoirs with surface structures are more likely to adhere to the intestinal mucosa in the correct orientation and thus realize unidirectional drug release toward it.

During the performance of the experiments it was observed that when placing the devices downward, the surface structures rapidly penetrated the mucus. In contrast to tensile mucoadhesion tests that apply vertical stress, the flow retention test induces shear forces acting laterally on the devices. The surface structures, by penetrating the mucus, presumably increase the contact area and lead to a mechanical interlocking that increases resistance against lateral shear stress. Additionally, penetration reduces the effective surface area subjected to the flow, compared to placing the minireservoirs upward.

3.3. Simulation of Shear Stress/Drag. Computer simulations relying on a simplified understanding of intestinal flow, in which the surface structures are likewise penetrating the mucus (in this case the wall of a cylinder), show a difference in drag force for downward and upward orientations, when the devices are subjected to a laminar flow over the samerange of flow rates (Figure 5). The resulting asymmetry (orientation bias) in drag force versus flow rate for the different designs shows similarities to the asymmetry in the experimentally determined retention profiles of the devices. Acomparison of orientation biases as quotients of values obtained for downward and upward orientation between experimentally determined values for retention and simulated

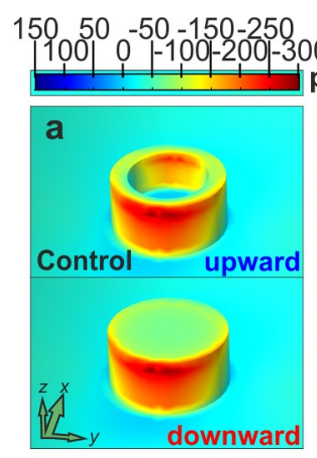

\section{pN}
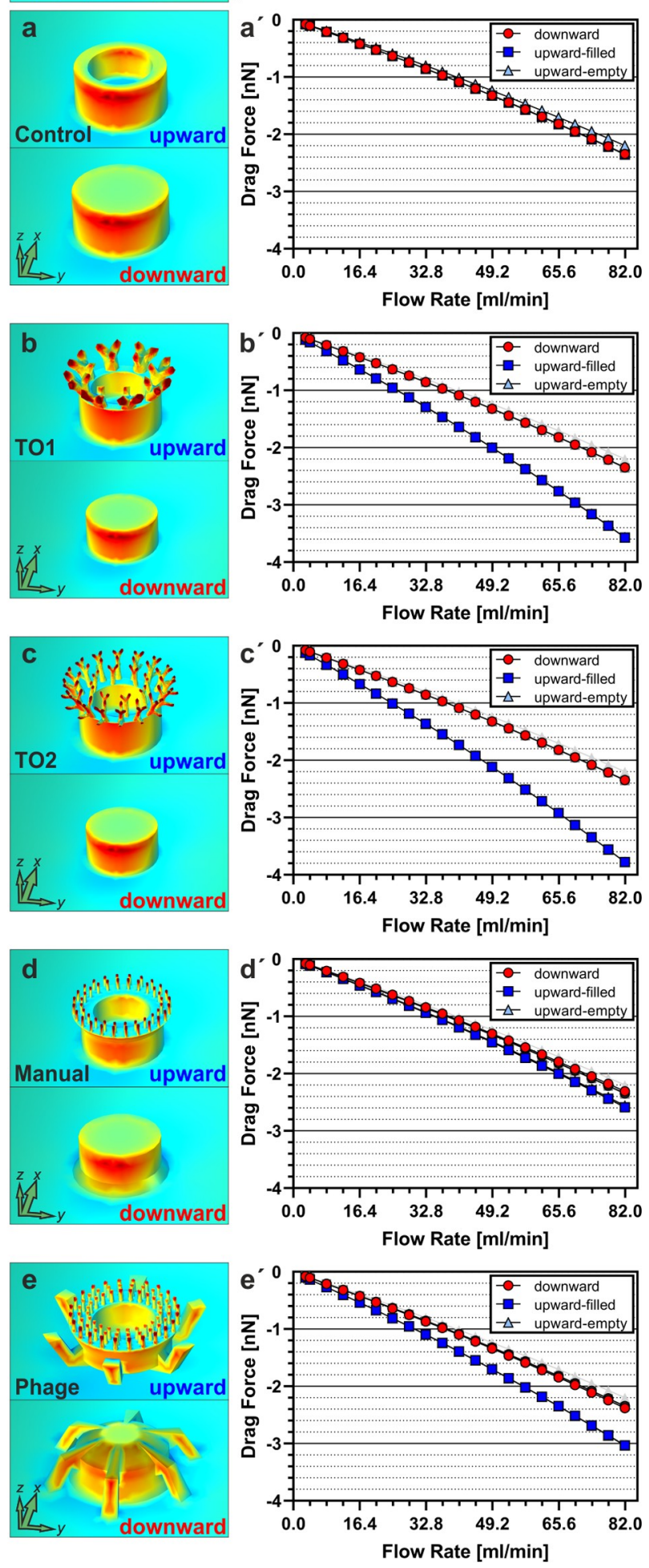

Figure 5. Simulation of drag on different reservoir geometries in different orientations under laminar flow ( $x$-direction) conditions in a tube $\left(5 \times 10 \mathrm{~cm}^{2}\right)$. When headed downward, surface structures are simulated to enter the wall to mimic penetration of mucus. $(\mathrm{a}-\mathrm{e})$ Surface plots of drag in the $x$ direction for different designs and $\left(\mathrm{a}^{\prime}-\right.$ $\left.\mathrm{e}^{\prime}\right)$ computed drag force plotted against different flow rates. Values from $\mathrm{a}^{\prime}$ grayed out in $\mathrm{b}^{\prime}$ to $\mathrm{e}^{\prime}$.

values for drag force, show a similar trend (si Figure S8a). Consequently, increased retention of minireservoirs may be 
due to a combination of mechanical mucoadhesion and reduced shear stress/drag.

3.4. Further Discussion. As of yet, reservoir devices for ODD were fabricated in sizes ranging from 50 to $300 \mu \mathrm{m} .{ }^{1,9}$ By presenting reservoir devices with diameters from 400 to 3600 $\mu \mathrm{m}$, this work drastically expands the size range of fabricated devices and calls into question whether there is a specific size range for optimal functionality. In this context it should be noted that larger devices are subjected to higher shear stress and drag than smaller ones. This would be an important design constraint for reservoir devices that are designed to have an orientation bias based on a difference in shear stress/drag. However, computer simulations of drag force acting on reservoir devices with different sizes show only a marginal effect of size on orientation bias (SI Figure S8b). Another important parameter to be considered is the drug loading efficiency of reservoir devices. A reduction of fabrication limitations at elevated dimensions can lead to a higher loading efficiency, e.g., through decreased relative wall-thicknesses (SI Figure S9).

With respect to the outlined fabrication scheme of micro/ minireservoir devices via 3D printing (Figure 1), it should be considered that $3 \mathrm{D}$ printed reservoir devices featuring surface structures, such as those presented in this work, might interfere with current postprocessing procedures like drug loading or lid sealing. It is unlikely that the surface structures have an impact on drug loading via inkjet printing as performed by Marizza et al., due to the needle/syringe having a clear vertical pathtoward the cavity of the reservoir devices as is the case with conventional cylindrical reservoir devices. ${ }^{20}$ However, a drug loading method based on the use of a shadow mask as demonstrated by Abid et al. could be impacted by aforementioned surface structures..$^{22}$ This is because the latter might hinder the shadow mask to come into contact with the reservoir edges and thus weaken the shadow mask's function to prevent drug powder from entering the interspace between the surface structures and the reservoir devices. Therefore, such a method would need to be optimized for the use with 3D printed reservoir devices featuring surface structures. This could be achieved by employing a different kind of shadow mask, e.g., a shadow mask exhibiting funnel-like structures that restrict any powder filling to the cavities of the reservoirdevices (SI Figure S10). In previous studies, microreservoirs have been sealed with a $\mathrm{pH}$-sensitive sealant polymer that dissolves at $\mathrm{pH}$ values present in the intestine. 6,9 In these cases, the sealant polymer was applied to the microreservoirs using a spray coater. It is expected that any surface structures on $3 \mathrm{D}$ printed reservoir devices will be covered with sealant polymerif a similar lid sealing process via spray coating is employed. Depending on the dissolution rate of the sealant polymer in the intestinal environment, the mucoadhesive effect of the surface structures during application of the reservoir devices could be inhibited or decreased. Moreover, the surface structures might prevent the sealant polymer from effectively sealing the cavity of the reservoir devices, thus potentially allowing premature leakage of drug. As it remains unclear howthe surface structures will affect the mentioned postprocessingprocedures and the drug release from the $3 \mathrm{D}$ printed reservoirdevices, further investigations are required in the future.

\section{CONCLUSIONS}

We have demonstrated the implementation of $3 \mathrm{D}$ printing into potential workflows for ODD microdevice fabrication by $3 \mathrm{D}$ printing microreservoirs with different shapes and sizes on sacrificial release substrates. The suggested approach provides a cost-effective and flexible alternative for the fabrication of reservoir devices and is compatible with subsequent postprocessing steps (such as drug loading and sealing), which have been reported in the literature. Moreover, the approach is suitable for repeatable fabrication as well as fast iterations with changing parameters and designs.

To highlight the prototyping potential of 3D printing for the fabrication of ODD devices, we showed the design and fabrication of various minireservoirs featuring anchor-like surface structures for geometry-based mechanical mucoadhesion. Increased adhesiveness to the intestinal mucosa, with representative values up to a 2 -fold higher compared to a control, as well as implications for unidirectional release with controlled orientation toward the mucosal surface have been presented experimentally and theoretically. The results indicate that mucoadhesive surface structures can be regarded as a promising solution to enhance the mucoadhesive performance and thereby potentially the therapeutic effect of reservoir devices for oral drug delivery.

\section{ASSOCIATED CONTENT}

\section{Supporting Information}

The Supporting Information is available free of charge at https://pubs.acs.org/doi/10.1021/acsbiomaterials.9b01760.

Figure S1, Photograph of different micro-/mini-reservoir devices on sacrificial polyvinyl alcohol substrates and customized holder for 3D printer; Figure S2, scanning electron microscopy images of array of Control-design with different sizes; Figure S3, scanning electron microscopy images of array of TO1-design with different sizes; Figure S4, scanning electron microscopy images of array of TO2-design with different sizes; Figure S5, scanning electron microscopy images of array of bioinspired Phage-design (without spikes) with different sizes; Figure S6, scanning electron microscopy images of $3 \mathrm{D}$ printed array of miniaturized reservoir devices with edge anchors (TO1) on sacrificial polyvinyl alcohol (PVA) substrates; Figure S7, tensile mucoadhesion measurement; Figure S8, comparison of orientation bias of different reservoir designs; Figure S9, drug to nondrug material ratio of one mini-reservoir and SU8 microcontainers; Figure S10, potential adaptation of powder embossing method for drug loading of microcontainers; and additional references (PDF)

\section{AUTHOR INFORMATION}

\section{Corresponding Authors}

Lukas Vaut - The Danish National Research Foundation and Villum Foundation's Center for Intelligent Drug Delivery and Sensing Using Microcontainers and Nanomechanics (IDUN), Department of Health Technology, Technical University of Denmark, Kongens Lyngby 2800, Denmark; ○ orcid.org/ 0000-0002-8857-4943; Email: lukv@dtu.dk

Anja Boisen - The Danish National Research Foundation and Villum Foundation's Center for Intelligent Drug Delivery and Sensing Using Microcontainers and Nanomechanics (IDUN), Department of Health Technology, Technical University of Denmark, Kongens Lyngby 2800,Denmark; Email: aboi@, dtu.dk 


\section{Authors}

Julia J. Juszczyk - The Danish National Research Foundation and Villum Foundation's Center for Intelligent Drug Delivery and Sensing Using Microcontainers and Nanomechanics (IDUN), Department of Health Technology, Technical University of Denmark, Kongens Lyngby 2800, Denmark Khorshid Kamguyan - The Danish National Research Foundation and Villum Foundation's Center for Intelligent Drug Delivery and Sensing Using Microcontainers and Nanomechanics (IDUN), Department of Health Technology, Technical University of Denmark, Kongens Lyngby 2800, Denmark

Kristian E. Jensen - The Danish National Research Foundation and Villum Foundation's Center for Intelligent Drug Delivery and Sensing Using Microcontainers and Nanomechanics (IDUN), Department of Health Technology, Technical University of Denmark, Kongens Lyngby 2800, Denmark

Guido Tosello - Department of Mechanical Engineering, Technical University of Denmark, Kongens Lyngby 2800, Denmark

Complete contact information is available at: https://pubs.acs.org/10.1021/acsbiomaterials.9b01760

\section{Author Contributions}

The manuscript was written through contributions of all authors. All authors have given approval to the final version of the manuscript.

\section{Funding}

Danish National Research Foundation (DNRF122) and Villum Foundation (Grant No. 9301).

\section{Notes}

The authors declare no competing financial interest.

\section{ACKNOWLEDGMENTS}

The authors would like to acknowledge the Center for Intelligent Drug Delivery and Sensing Using Microcontainers and Nanomechanics (IDUN) whose research is funded by the Danish National Research Foundation (DNRF122) and Villum Foundation (Grant No. 9301). The authors wouldalso like to thank Tommy Sonne Alstrøm and Kinga Zorfor help with the preparation of the manuscript as well as Jesper Scheel for photography.

\section{REFERENCES}

(1) Ahmed, A.; Bonner, C.; Desai, T. A. Bioadhesive Microdevices for Drug Delivery: A Feasibility Study. Biomed. Microdevices 2001, 3 (2), 89-96.

(2) Nielsen, L. H.; Keller, S. S.; Boisen, A. Microfabricated Devices for Oral Drug Delivery. Lab Chip 2018, 18 (16), 2348-2358.

(3) Anselmo, A. C.; Gokarn, Y.; Mitragotri, S. Non-Invasive Delivery Strategies for Biologics. Nat. Rev. Drug Discovery 2019, 18 (1), 19-40.

(4) Tao, S. L.; Desai, T. A. Microfabrication of Multilayer, Asymmetric, Polymeric Devices for Drug Delivery. Adv. Mater. 2005, 17 (13), 1625-1630.

(5) Petersen, R. S.; Keller, S. S.; Boisen, A. Hot Punching of HighAspect-Ratio 3D Polymeric Microstructures for Drug Delivery. Lab Chip 2015, 15 (12), 2576-2579.

(6) Nielsen, L. H.; Melero, A.; Keller, S. S.; Jacobsen, J.; Garrigues, T.; Rades, T.; Mullertz, A.; Boisen, A. Polymeric Microcontainers Improve Oral Bioavailability of Furosemide. Int. J. Pharm. 2016, 504 $(1-2), 98-109$.

(7) McHugh, K. J.; Nguyen, T. D.; Linehan, A. R.; Yang, D.; Behrens, A. M.; Rose, S.; Tochka, Z. L.; Tzeng, S. Y.; Norman, J. J.; Anselmo, A. C.; Xu, X.; Tomasic, S.; Taylor, M. A.; Lu, J.; Guarecuco,
R.; Langer, R.; Jaklenec, A. Fabrication of Fillable Microparticles and Other Complex 3D Microstructures. Science (Washington, DC, U. S.) 2017, 357 (6356), 1138-1142.

(8) Ainslie, K. M.; Kraning, C. M.; Desai, T. A. Microfabrication of an Asymmetric, Multi-Layered Microdevice for Controlled Release of Orally Delivered Therapeutics. Lab Chip 2008, 8 (7), 1042-1047.

(9) Mazzoni, C.; Tentor, F.; Strindberg, S. A.; Nielsen, L. H.; Keller, S. S.; Alstrøm, T. S.; Gundlach, C.; Müllertz, A.; Marizza, P.; Boisen, A. From Concept to in Vivo Testing: Microcontainers for Oral Drug Delivery. J. Controlled Release 2017, 268, 343-351.

(10) Ahmed, A.; Bonner, C.; Desai, T. A. Bioadhesive Microdevices with Multiple Reservoirs: A New Platform for Oral Drug Delivery. J. Controlled Release 2002, 81 (3), 291-306.

(11) Chirra, H. D.; Shao, L.; Ciaccio, N.; Fox, C. B.; Wade, J. M.; Ma, A.; Desai, T. A. Planar Microdevices for Enhanced In Vivo Retention and Oral Bioavailability of Poorly Permeable Drugs. Adv. Healthcare Mater. 2014, 3 (10), 1648-1654.

(12) Quinlan, H. E.; Hasan, T.; Jaddou, J.; Hart, A. J. Industrial and Consumer Uses of Additive Manufacturing: A Discussion of Capabilities, Trajectories, and Challenges. J. Ind. Ecol. 2017, 21 (0), S15-S20.

(13) Okwuosa, T. C.; Pereira, B. C.; Arafat, B.; Cieszynska, M.; Isreb, A.; Alhnan, M. A. Fabricating a Shell-Core Delayed Release Tablet Using Dual FDM 3D Printing for Patient-Centred Therapy. Pharm. Res. 2017, 34 (2), 427-437.

(14) Beck, R. C. R.; Chaves, P. S.; Goyanes, A.; Vukosavljevic, B.; Buanz, A.; Windbergs, M.; Basit, A. W.; Gaisford, S. 3D Printed Tablets Loaded with Polymeric Nanocapsules: An Innovative Approach to Produce Customized Drug Delivery Systems. Int. J. Pharm. 2017, 528 (1-2), 268-279.

(15) Vaezi, M.; Seitz, H.; Yang, S. A Review on 3D Micro-Additive Manufacturing Technologies. Int. J. Adv. Manuf. Technol. 2013, 67 (5-8), 1721-1754.

(16) Vaut, L.; Jensen, K. E.; Tosello, G.; Khosla, A.; Furukawa, H.; Boisen, A. Additive Manufacturing of Microreservoir Devices for Oral Drug Delivery Using an Acculas BA-30 Micro-Stereolithography Instrument: A Feasibility Study. J. Electrochem. Soc. 2019, 166 (9), B3257-B3263

(17) Vaut, L.; Zeng, G.; Tosello, G.; Boisen, A. Sacrificial Polymer Substrates in Photopolymerization-Based Micro 3D Printing for Fabrication and Release of Complex Micro Components. Adv. Mater. Technol. 2019, 4, 1900378.

(18) Ahluwalia, N. K.; Thompson, D. G.; Barlow, J.; Heggie, L. Human Small Intestinal Contractions and Aboral Traction Forces during Fasting and after Feeding. Gut 1994, 35 (5), 625-630.

(19) Mudie, D. M.; Amidon, G. L.; Amidon, G. E. Physiological Parameters for Oral Delivery and in Vitro Testing. Mol. Pharmaceutics 2010, 7 (5), 1388-1405.

(20) Marizza, P.; Keller, S. S.; Boisen, A. Inkjet Printing as a Technique for Filling of Micro-Wells with Biocompatible Polymers. Microelectron. Eng. 2013, 111, 391-395.

(21) Fox, C. B.; Nemeth, C. L.; Chevalier, R. W.; Cantlon, J.; Bogdanoff, D. B.; Hsiao, J. C.; Desai, T. A. Picoliter-Volume Inkjet Printing into Planar Microdevice Reservoirs for Low-Waste, HighCapacity Drug Loading. Bioeng. Transl. Med. 2017, 2 (1), 9-16.

(22) Abid, Z.; Gundlach, C.; Durucan, O.; von Halling Laier, C.; Nielsen, L. H.; Boisen, A.; Keller, S. S. Powder Embossing Method for Selective Loading of Polymeric Microcontainers with Drug Formulation. Microelectron. Eng. 2017, 171, 20-24.

(23) Nielsen, L. H.; Nagstrup, J.; Gordon, S.; Keller, S. S.; Østergaard, J.; Rades, T.; Müllertz, A.; Boisen, A. PH-Triggered Drug Release from Biodegradable Microwells for Oral Drug Delivery. Biomed. Microdevices 2015, 17 (3), 1-7.

(24) Naisbett, B.; Woodley, J. The Potential Use of Tomato Lectin for Oral Drug Delivery. 1. Lectin Binding to Rat Small Intestine in Vitro. Int. J. Pharm. 1994, 107 (3), 223-230.

(25) Smart, J. D. The Basics and Underlying Mechanisms of Mucoadhesion. Adv. Drug Delivery Rev. 2005, 57 (11), 1556-1568. 
(26) Jensen, K. E. Solving 2D/3D Heat Conduction Problems by Combining Topology Optimization and Anisotropic Mesh Adaptation. In Advances in Structural and Multidisciplinary Optimization;

Springer International Publishing: Cham, 2018; pp 1224-1238 DOI: 10.1007/978-3-319-67988-4_92.

(27) Hu, B.; Margolin, W.; Molineux, I. J.; Liu, J. Structural Remodeling of Bacteriophage T4 and Host Membranes during Infection Initiation. Proc. Natl. Acad. Sci. U. S. A. 2015, 112 (35), E4919-E4928.

(28) Cone, R. A. Barrier Properties of Mucus. Adv. Drug Delivery Rev. 2009, 61 (2), 75-85.

(29) Edsman, K.; Hägrström, H. Pharmaceutical Applications of Mucoadhesion for the Non-Oral Routes. J. Pharm. Pharmacol. 2005, 57 (1), 3-22.

(30) Rao, K. V. R.; Buri, P. A Novel in Situ Method to Test Polymers and Coated Microparticles for Bioadhesion. Int. J. Pharm. 1989, 52 (3), 265-270.

(31) Kerlin, P.; Zinsmeister, A.; Phillips, S. Relationship of Motility to Flow of Contents in the Human Small Intestine. Gastroenterology 1982, 82 (4), 701-706. 